Analele Universităţii de Vest, Timişoara

Seria Matematică - Informatică

LIV, 1, (2016), 139- 157

\title{
Higher-Order Geodesic Equations from Non-Local Lagrangians and Complex Backward-Forward Derivative Operators
}

\author{
Rami Ahmad El-Nabulsi
}

\begin{abstract}
Starting with an extended complex backwardforward derivative operator in differential geometry which is motivated from non-local-in-time Lagrangian dynamics, higher-order geodesic equations are obtained within classical differential geometrical settings. We limit our analysis up to the $2^{\text {nd }}$-order derivative where some applications are discussed and a number of features are revealed accordingly.
\end{abstract}

AMS Subject Classification (2000). 53C22; 37N20

Keywords. Non-local-in-time Lagrangians; Extended complex backward-forward derivative operator; higher-order geodesic equations; higher-order geodesic deviation equations; complexified metric.

\section{Introduction}

In differential geometry and particularly in Riemannian metric space, geodesics are the shortest path between points in space. They play an important role in general relativity, in spacetime curvature and the two-body problem mainly the perihelion advance for the planet Mercury which is based on the solution of the geodesic equation in the Schwarzschild spacetime metric [?14]. 
However, generalizations of geodesics to higher-order derivatives were discussed in literature through different contexts, e.g. the study of the uniformly accelerated relativistic test particle world line [?9] and geodesic circle in pseudo-Riemannian geometry [?33] which involves $3^{\text {rd }}$-order derivatives $[? 14, ? 15]$; the study of the problem of closed orbital motion in the Kerr metric spacetime [?3]; the study of higher-order geodesics in Lie groups [?24] among others. In most of these works, higher-order geodesics are obtained by means of the geodesic deviation equation commonly known as the Jacob equation which relates the Riemann curvature tensor to the relative acceleration of two closed geodesics [?28] or by means of geodesic deviation at higher-order [?32].

In this paper, we would like to address the problem differently. We will prove that higher-order geodesics may be obtained by means of a generalized complex backward-forward derivative operator motivated from non-local-intime Lagrangian dynamics. This kind of dynamics was addressed long time ago in theoretical physics. In fact, Nelson for the $1^{\text {st }}$ time used non-localin-time approach to derive the Schrödinger equation starting from classical mechanical framework [?17].

Non-local-in-time dynamics was addressed recently in [?30] by introducing the notion of the non-local-in-in time kinetic energy. The topic was also touched in [?13] to deal with dissipative systems and in [?5] to study the case of deformed or non-standard Lagrangians type. Related issues concern scale relativity using concurrently the notion of backward-forward motion [?1,?19] on non-differentiable curves [?20] by means of a complex backward-forward derivative operator.

More specifically, in this paper we will extend the notion of the complex derivative operator for the case of non-local-in-time geometric Lagrangian dynamics and we will prove that within the classical differential geometrical settings, the classical Lagrangian procedure will lead to higher-order geodesic equations by means of the Taylor expansion of derivative operators that might have interesting features. Through this paper we limit ourselves for the $2^{\text {nd }}$ order derivative.

The paper is organized as follows: in Sec. 2, we introduce the basic mathematical setups where we derive the higher-order Euler-Lagrange equations and the higher-order geodesics; in Sec. 3 we illustrate by discussing the geometric dynamics of some specific cases; conclusions are given in Sec. 4. 
2 Basic Setups: Non-local-in-time Coordinates, Lagrangians, the Higher-order Euler-Lagrange Equations and the Higher-order Geodesics

We start by introducing the basic setups of our approach.

Definition 2.1. Given a dynamical system characterized by a generalized coordinate $\mathbf{X}$, the respectively backward and forward trajectories $\mathbf{X}(t-\bar{\tau})$ and $\mathbf{X}(t+\bar{\tau}) ; t$ being the proper time and $\bar{\tau}$ a positive constant. The complex backward-forward derivative operator $\mathbf{D}$ of $\mathbf{X}$ is defined by [?19]:

$$
\begin{array}{r}
\mathbf{D X} \triangleq \frac{1}{2}\left(\frac{d \mathbf{X}(t+\bar{\tau})}{d t}+\frac{d \mathbf{X}(t-\bar{\tau})}{d t}\right)-\frac{i}{2}\left(\frac{d \mathbf{X}(t+\bar{\tau})}{d t}-\frac{d \mathbf{X}(t-\bar{\tau})}{d t}\right) \\
\equiv \frac{1-i}{2} D \mathbf{X}(t+\bar{\tau})+\frac{1+i}{2} D \mathbf{X}(t-\bar{\tau})
\end{array}
$$

where $i=\sqrt{-1} \in C$ and $D=d / d t$.

In fact, the operator $\mathbf{D}$ was introduced in scale relativity [?19] which describes the fractal spacetime theory that allows recuperating local differential time reversibility in terms of a new complex process [?20]. We can extend this operator using the following Taylor series expansions:

$$
\begin{aligned}
\mathbf{X}(t+\bar{\tau}) & \approx \mathbf{X}(t)+\bar{\tau} D \mathbf{X}(t)+\frac{1}{2 !} \bar{\tau}^{2} D^{2} \mathbf{X}(t)+\ldots+\frac{1}{n !} \bar{\tau}^{n} D^{(n)} \mathbf{X}(t) \\
& =\mathbf{X}(t)+\sum_{k=1}^{n} \frac{1}{k !} \bar{\tau}^{k} D^{(k)} \mathbf{X}(t) \\
\mathbf{X}(t-\bar{\tau}) & \approx \mathbf{X}(t)-\bar{\tau} D \mathbf{X}(t)+\frac{1}{2 !} \bar{\tau}^{2} D^{2} \mathbf{X}(t)+\ldots+\frac{(-1)^{n}}{n !} \bar{\tau}^{n} D^{(n)} \mathbf{X}(t) \\
& =\mathbf{X}(t)+\sum_{k=1}^{n} \frac{(-1)^{k}}{k !} \bar{\tau}^{k} D^{(k)} \mathbf{X}(t)
\end{aligned}
$$

Lemma 2.1. Using equations (2.1) and (2.2), the extended complex backwardforward derivative operator acting on $\mathbf{X}(t)$ takes the form:

$$
\begin{aligned}
\mathbf{D}^{n} \mathbf{X}(t)= & \frac{1-i}{2}\left(D \mathbf{X}(t)+\sum_{k=1}^{n} \frac{1}{k !} \bar{\tau}^{k} D^{(k+1)} \mathbf{X}(t)\right) \\
& +\frac{1+i}{2}\left(D \mathbf{X}(t)+\sum_{k=1}^{n} \frac{(-1)^{k}}{k !} \bar{\tau}^{k} D^{(k+1)} \mathbf{X}(t)\right)
\end{aligned}
$$


$=D \mathbf{X}(t)+\frac{1}{2} \sum_{k=1}^{n}\left(1+(-1)^{k}\right) \frac{1}{k !} \bar{\tau}^{k} D^{(k+1)} \mathbf{X}(t)-\frac{i}{2} \sum_{k=1}^{n}\left(1-(-1)^{k}\right) \frac{1}{k !} \bar{\tau}^{k} D^{(k+1)} \mathbf{X}(t)$

In order to generalize this operator for the case of non-local-in-time process, we introduce the following definition:

Definition 2.2. We define the non-local-in-time generalized coordinates by:

$$
\begin{gathered}
\mathbf{Y}_{\bar{\tau}}^{n}=\frac{\mathbf{X}(t+\bar{\tau})+\mathbf{X}(t-\bar{\tau})}{2}-i \frac{\mathbf{X}(t+\bar{\tau})-\mathbf{X}(t-\bar{\tau})}{2} \\
\equiv \mathbf{X}(t)+\frac{1}{2}\left(\sum_{k=1}^{n} \frac{1}{k !}\left(1+(-1)^{k}\right) \bar{\tau}^{k} D^{(k)} \mathbf{X}(t)-i \sum_{k=1}^{n} \frac{1}{k !}\left(1-(-1)^{k}\right) \bar{\tau}^{k} D^{(k)} \mathbf{X}(t)\right) .
\end{gathered}
$$

Definition 2.3. The extended complex backward-forward derivative operator action of $Y_{\tau}^{n}$ is defined accordingly by:

$$
\begin{gathered}
\mathbf{D}^{n}=D+\underbrace{\frac{1}{2} \sum_{k=1}^{n}\left(1+(-1)^{k}\right) \frac{1}{k !} \bar{\tau}^{k} D^{(k+1)}}_{\mathcal{D}_{R}^{n}}-i \underbrace{i \frac{1}{2} \underbrace{n}_{k=1}\left(1-(-1)^{k}\right) \frac{1}{k !} \bar{\tau}^{k} D^{(k+1)}}_{\mathcal{D}_{I}^{n}}, \\
\equiv D+\mathcal{D}_{R}^{n}-i \mathcal{D}_{I}^{n} .
\end{gathered}
$$

Evidently, for $n=1$, we find $\mathbf{Y}_{\tau}^{1}=\mathbf{X}(t)-i \bar{\tau} \dot{\mathbf{X}}(t)$ and $\mathbf{D}^{1}=D-i \bar{\tau} D^{(2)}$. As a result we get effortlessly $\mathbf{D}^{1} \mathbf{Y}_{\tau}^{1}=\left(D-i \bar{\tau} D^{(2)}\right)(\mathbf{X}(t)-i \bar{\tau} \dot{\mathbf{X}}(t))=\dot{\mathbf{X}}(t)-$ $2 i \bar{\tau} \ddot{\mathbf{X}}(t)-\bar{\tau}^{2}(t)$.

We can now give the necessary condition for the extremum of a function of type $S=\int_{a}^{b} L_{n}\left(\mathbf{D}^{n} \mathbf{Y}_{\bar{\tau}}^{n}, \mathbf{Y}_{\bar{\tau}}^{n}, t\right) d t$ where the curves are allowed to vary between two fixed points. Here $\mathbf{Y}_{\bar{\tau}}^{n} \in C^{1}[a, b]$ and $L_{n}\left(\mathbf{D}^{n} \mathbf{Y}_{\bar{\tau}}^{n}, \mathbf{Y}_{\bar{\tau}}^{n}, t\right) \in$ $C^{2}\left([a, b] \times C^{n} \times C^{n} ; C\right)$ is a function with continuous derivatives with respect to $\left(\mathbf{D}^{n} \mathbf{Y}_{\bar{\tau}}^{n}, \mathbf{Y}_{\bar{\tau}}^{n}, t\right)$. The problem is to find $\mathbf{Y}_{\bar{\tau}}^{n} \in C^{1}[a, b]$ such that $\mathbf{Y}_{\bar{\tau}}^{n}(a)=$ $\mathbf{y}(a), \mathbf{Y}_{\bar{\tau}}^{n}(b)=\mathbf{y}(b)$ and which is an extremum of $S=\int_{a}^{b} L_{n}\left(\mathbf{D}^{n} \mathbf{Y}_{\bar{\tau}}^{n}, \mathbf{Y}_{\bar{\tau}}^{n}, t\right) d t$.

Theorem 2.1. If the action functional $S=\int_{a}^{b} L_{n}\left(\mathbf{D}^{n} \mathbf{Y}_{\bar{\tau}}^{n}, \mathbf{Y}_{\bar{\tau}}^{n}, t\right) d t$ has an extremum at $\mathbf{Y}_{\bar{\tau}}^{n} \in \mathbf{S}=\left\{\mathbf{Y}_{\bar{\tau}}^{n} \in C^{1}[a, b] ; \mathbf{Y}_{\bar{\tau}}^{n}(a)=\mathbf{y}(a), \mathbf{Y}_{\bar{\tau}}^{n}(b)=\mathbf{y}(b)\right\}$, then the following higher-order Euler-Lagrange equations hold [?25]:

$$
\frac{\partial L_{n}\left(\mathbf{D}^{n} \mathbf{Y}_{\bar{\tau}}^{n}, \mathbf{Y}_{\bar{\tau}}^{n}, t\right)}{\partial \mathbf{Y}_{\bar{\tau}}^{n}}-\mathbf{D}^{n}\left(\frac{\partial L_{n}\left(\mathbf{D}^{n} \mathbf{Y}_{\bar{\tau}}^{n}, \mathbf{Y}_{\bar{\tau}}^{n}, t\right)}{\partial \mathbf{D}^{n} \mathbf{Y}_{\bar{\tau}}^{n}}\right)=0
$$


It is obvious that for $n=1$, this equation is reduced to the $2^{\text {nd }}$-order derivative Euler-Lagrange equation:

$\frac{\partial L_{1}\left(\mathbf{D}^{1} \mathbf{Y}_{\bar{\tau}}^{1}, \mathbf{Y}_{\bar{\tau}}^{1}, t\right)}{\partial \mathbf{Y}_{\bar{\tau}}^{1}}-\frac{d}{d t}\left(\frac{\partial L_{1}\left(\mathbf{D}^{1} \mathbf{Y}_{\bar{\tau}}^{1}, \mathbf{Y}_{\bar{\tau}}^{1}, t\right)}{\partial \mathbf{D}^{1} \mathbf{Y}_{\bar{\tau}}^{1}}\right)+i \bar{\tau} \frac{d^{2}}{d t^{2}}\left(\frac{\partial L_{1}\left(\mathbf{D}^{1} \mathbf{Y}_{\bar{\tau}}^{1}, \mathbf{Y}_{\bar{\tau}}^{1}, t\right)}{\partial \mathbf{D}^{1} \mathbf{Y}_{\bar{\tau}}^{1}}\right)=0$

It is well-known that the Lagrangian formalism of classical mechanics may be translated in curved spacetime with line element $d s^{2}=g_{\alpha \beta} d x^{\alpha} d x^{\beta}, \alpha, \beta=$ $0,1,2,3, g_{\alpha \beta}$ is the metric.

In our arguments, we have $d \mathbf{s}^{2}=\mathbf{g}_{\alpha \beta} \mathbf{D}^{n} \mathbf{Y}_{\bar{\tau}}^{n^{\alpha}} \mathbf{D}^{n} \mathbf{Y}_{\bar{\tau}}^{n^{\beta}}, \alpha, \beta=0,1,2,3$. Here $\mathbf{g}_{\alpha \beta}$ is a complexified metric. We assume in what follows $c=1$, cis the celerity of light. In what follows, we will restrict ourselves for $n=1$. We consider an $N$-dimensional manifold $\mathcal{M}$, a parameterized curve $\Upsilon:[a, b] \rightarrow \mathcal{M}$ and a parameter $\sigma \in[a, b]$ such that spacetime coordinates are given by the parameterized worldline $Y^{\alpha}(\sigma)$. In order to derive the higher-order geodesic equations, we use the classical variational principle in curved geometry which states that freely falling test particles follow a path between two fixed points $A(a)$ and $B(b)$ in spacetime which extremizes the proper time $\tau$ defined by $d \tau^{2}=-d \mathbf{s}^{2}[? 6, ? 8]$. In order to apply the Lagrangian formalism, we set $\sigma=0$ at $A$ and $\sigma=1$ at $B$. So, we can write $\tau=\int_{0}^{1} \sqrt{-\mathbf{g}_{\alpha \beta} \mathbf{D}^{1} \mathbf{Y}_{\bar{\tau}}^{{ }^{\alpha}} \mathbf{D}^{1} \mathbf{Y}_{\bar{\tau}}^{1^{\beta}}} d \sigma \equiv$ $\int_{0}^{1} L_{1}\left(\mathbf{D}^{1} \mathbf{Y}_{\bar{\tau}}^{1^{\alpha}}, \mathbf{Y}_{\bar{\tau}}^{1^{\alpha}}\right) d \sigma$ where $L_{1}\left(\mathbf{D}^{1} \mathbf{Y}_{\bar{\tau}}^{1^{\alpha}}, \mathbf{Y}_{\bar{\tau}}^{1^{\alpha}}\right) \triangleq L_{1}=d \tau / d \sigma$ and accordingly the Euler-Lagrange equation that we will use takes the form:

$$
\frac{\partial L_{1}}{\partial \mathbf{Y}_{\bar{\tau}}^{1 \gamma}}-\mathbf{D}^{1}\left(\frac{\partial L_{1}}{\partial \mathbf{D}^{1} \mathbf{Y}_{\bar{\tau}}^{1 \gamma}}\right)=0
$$

where $\mathbf{D}^{1}=D-i \bar{\tau} D^{(3)}$. Given a function $f=f(\tau(\sigma))$, then the following relations hold:

$$
\begin{gathered}
\frac{d f}{d \sigma}=L_{1} \frac{d f}{d \tau} \\
\frac{d^{2} f}{d \sigma^{2}}=\frac{d}{d \sigma}\left(\frac{d f}{d \sigma}\right)=\frac{d}{d \sigma}\left(L_{1} \frac{d f}{d \tau}\right)=\frac{d L_{1}}{d \sigma} \frac{d f}{d \tau}+L_{1}^{2} \frac{d^{2} f}{d \tau^{2}} \\
\frac{d^{3} f}{d \sigma^{3}}=\frac{d^{2} L_{1}}{d \sigma^{2}} \frac{d f}{d \tau}+L_{1} \frac{d L_{1}}{d \sigma} \frac{d^{2} f}{d \tau^{2}}+2 L_{1} \frac{d L_{1}}{d \sigma} \frac{d^{2} f}{d \tau^{2}}+L_{1}^{3} \frac{d^{3} f}{d \tau^{3}}
\end{gathered}
$$

Theorem 2.2. The higher-order geodesic equation for

$$
\tau=\int_{0}^{1} \sqrt{-\mathbf{g}_{\alpha \beta} \mathbf{D}^{1} \mathbf{Y}_{\bar{\tau}}^{1^{\alpha}} \mathbf{D}^{1} \mathbf{Y}_{\bar{\tau}}^{1 \beta}} d \sigma \equiv \int_{0}^{1} L_{1}\left(\mathbf{D}^{1} \mathbf{Y}_{\bar{\tau}}^{1^{\alpha}}, \mathbf{Y}_{\bar{\tau}}^{1^{\alpha}}\right) d \sigma
$$


is given by:

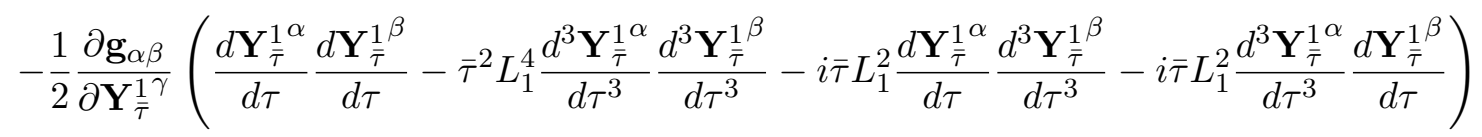

$$
\begin{aligned}
& +\mathbf{g}_{\alpha \gamma} \frac{d^{2} \mathbf{Y}_{\bar{\tau}}^{1^{\alpha}}}{d \tau^{2}}+\frac{1}{2}\left(\frac{\partial \mathbf{g}_{\alpha \gamma}}{\partial \mathbf{Y}_{\bar{\tau}}^{1}{ }^{\beta}}+\frac{\partial \mathbf{g}_{\gamma \beta}}{\partial \mathbf{Y}_{\bar{\tau}}^{1}}\right) \frac{d \mathbf{Y}_{\bar{\tau}}^{1}}{d \tau} \frac{d \mathbf{Y}_{\bar{\tau}}^{1}{ }^{\beta}}{d \tau} \\
& -i \bar{\tau} L_{1}^{2}\left(\mathbf{g}_{\alpha \gamma} \frac{d^{4} \mathbf{Y}_{\bar{\tau}}^{1}{ }^{\alpha}}{d \tau^{4}}+\frac{1}{2}\left(\frac{\partial \mathbf{g}_{\alpha \gamma}}{\partial \mathbf{Y}_{\bar{\tau}}^{1 \beta}}+\frac{\partial \mathbf{g}_{\gamma \beta}}{\partial \mathbf{Y}_{\bar{\tau}}^{1}{ }^{\alpha}}\right) \frac{d^{3} \mathbf{Y}_{\bar{\tau}}^{1}{ }^{\alpha}}{d \tau^{3}} \frac{d \mathbf{Y}_{\bar{\tau}}^{1 \beta}}{d \tau}\right) \\
& -i \bar{\tau} L_{1}^{2}\left(\mathbf{g}_{\alpha \gamma} \frac{d^{4} \mathbf{Y}_{\overline{\bar{\tau}}}^{{ }^{\alpha}}}{d \tau^{4}}+\frac{1}{2}\left(\frac{\partial \mathbf{g}_{\alpha \gamma}}{\partial \mathbf{Y}_{\bar{\tau}}^{1^{\beta}}}+\frac{\partial \mathbf{g}_{\gamma \beta}}{\partial \mathbf{Y}_{\bar{\tau}}^{1^{\alpha}}}\right) \frac{d \mathbf{Y}_{\bar{\tau}}^{1^{\beta}}}{d \tau} \frac{d^{3} \mathbf{Y}_{\overline{\bar{\tau}}}^{{ }^{\alpha}}}{d \tau^{3}}\right. \\
& +\frac{1}{2}\left(\frac{\partial \mathbf{g}_{\alpha \gamma}}{\partial \mathbf{Y}_{\bar{\tau}}^{1 \beta}}+\frac{\partial \mathbf{g}_{\gamma \beta}}{\partial \mathbf{Y}_{\bar{\tau}}^{1 \alpha}}\right)\left(\frac{d \mathbf{Y}_{\bar{\tau}}^{1 \beta}}{d \tau} \frac{d^{3} \mathbf{Y}_{\bar{\tau}}^{1}{ }^{1}}{d \tau^{3}}+2 \frac{d^{2} \mathbf{Y}_{\bar{\tau}}^{1}}{d \tau^{2}} \frac{d^{2} \mathbf{Y}_{\bar{\tau}}^{1^{\beta}}}{d \tau^{2}}+\frac{d \mathbf{Y}_{\bar{\tau}}^{1}}{d \tau} \frac{d^{3} \mathbf{Y}_{\bar{\tau}}^{1 \beta}}{d \tau^{3}}\right) \\
& \left.+\frac{1}{2}\left(2 \frac{d \mathbf{Y}_{\bar{\tau}}^{1}{ }^{\beta}}{d \tau} \frac{d^{2} \mathbf{Y}_{\bar{\tau}}^{1}}{d \tau^{2}}+\frac{d \mathbf{Y}_{\bar{\tau}}^{1}}{d \tau} \frac{d^{2} \mathbf{Y}_{\bar{\tau}}^{1 \beta}}{d \tau^{2}}\right)\left(\frac{\partial^{2} \mathbf{g}_{\alpha \gamma}}{\partial \mathbf{Y}_{\bar{\tau}}^{1 \beta 2}} \frac{d \mathbf{Y}_{\bar{\tau}}^{1 \beta}}{d \tau}+\frac{\partial^{2} \mathbf{g}_{\gamma \beta}}{\partial \mathbf{Y}_{\bar{\tau}}^{1^{\alpha}}} \frac{d \mathbf{Y}_{\bar{\tau}}^{1}}{d \tau}\right)\right) \\
& -\bar{\tau}^{2} L_{1}^{4}\left(\mathbf{g}_{\alpha \gamma} \frac{d^{6} \mathbf{Y}_{\bar{\tau}}^{1^{\alpha}}}{d \tau^{6}}+\frac{1}{2}\left(\frac{\partial \mathbf{g}_{\alpha \gamma}}{\partial \mathbf{Y}_{\bar{\tau}}^{1^{\beta}}}+\frac{\partial \mathbf{g}_{\gamma \beta}}{\partial \mathbf{Y}_{\bar{\tau}}^{1^{\alpha}}}\right) \frac{d \mathbf{Y}_{\bar{\tau}}^{1}{ }^{\beta}}{d \tau} \frac{d^{5} \mathbf{Y}_{\bar{\tau}}^{{ }^{\alpha}}}{d \tau^{5}}\right. \\
& +\frac{1}{2}\left(\frac{\partial^{2} \mathbf{g}_{\alpha \gamma}}{\partial \mathbf{Y}_{\bar{\tau}}^{1 \beta 2}} \frac{d \mathbf{Y}_{\bar{\tau}}^{1 \beta}}{d \tau}+\frac{\partial^{2} \mathbf{g}_{\gamma \beta}}{\partial \mathbf{Y}_{\bar{\tau}}^{1}{ }^{\alpha 2}} \frac{d \mathbf{Y}_{\bar{\tau}}^{1}}{d \tau}\right)\left(2 \frac{d \mathbf{Y}_{\bar{\tau}}^{1}{ }^{\beta}}{d \tau} \frac{d^{4} \mathbf{Y}_{\bar{\tau}}^{1}{ }^{\alpha}}{d \tau^{4}}+\frac{d^{3} \mathbf{Y}_{\bar{\tau}}^{1}{ }^{\alpha}}{d \tau^{3}} \frac{d^{2} \mathbf{Y}_{\bar{\tau}}^{1^{\beta}}}{d \tau^{2}}\right) \\
& +\frac{1}{2}\left(\frac{\partial \mathbf{g}_{\alpha \gamma}}{\partial \mathbf{Y}_{\bar{\tau}}^{1}{ }^{\beta}}+\frac{\partial \mathbf{g}_{\gamma \beta}}{\partial \mathbf{Y}_{\bar{\tau}}^{1}}\right)\left(2 \frac{d^{5} \mathbf{Y}_{\bar{\tau}}^{1}}{d \tau^{5}} \frac{d \mathbf{Y}_{\bar{\tau}}^{1}{ }^{\beta}}{d \tau}+3 \frac{d^{4} \mathbf{Y}_{\bar{\tau}}^{1^{\alpha}}}{d \tau^{4}} \frac{d^{2} \mathbf{Y}_{\bar{\tau}}^{1}{ }^{\beta}}{d \tau^{2}}+\frac{d^{3} \mathbf{Y}_{\bar{\tau}}^{1}}{d \tau^{3}} \frac{d^{3} \mathbf{Y}_{\bar{\tau}}^{{ }^{\beta}}}{d \tau^{3}}\right) \\
& +\frac{1}{2}\left(\frac{\partial^{2} \mathbf{g}_{\alpha \gamma}}{\partial \mathbf{Y}_{\bar{\tau}}^{1}{ }^{\beta 2}} \frac{d \mathbf{Y}_{\bar{\tau}}^{{ }^{\beta}}}{d \tau}+\frac{\partial^{2} \mathbf{g}_{\gamma \beta}}{\partial \mathbf{Y}_{\bar{\tau}}^{1}{ }^{\alpha 2}} \frac{d \mathbf{Y}_{\bar{\tau}}^{1}}{d \tau}\right)\left(\frac{d^{4} \mathbf{Y}_{\bar{\tau}}^{1}{ }^{\alpha}}{d \tau^{4}} \frac{d \mathbf{Y}_{\bar{\tau}}^{1^{\beta}}}{d \tau}+\frac{d^{3} \mathbf{Y}_{\bar{\tau}}^{\alpha}}{d \tau^{3}} \frac{d^{2} \mathbf{Y}_{\bar{\tau}}^{1^{\beta}}}{d \tau^{2}}\right) \\
& +\frac{1}{2}\left(\frac{\partial^{2} \mathbf{g}_{\alpha \gamma}}{\partial \mathbf{Y}_{\bar{\tau}}^{1}{ }^{\beta 2}} \frac{d^{2} \mathbf{Y}_{\bar{\tau}}^{1 \beta}}{d \tau^{2}}+\frac{\partial^{3} \mathbf{g}_{\alpha \gamma}}{\partial \mathbf{Y}_{\bar{\tau}}^{1 \beta 3}}\left(\frac{d \mathbf{Y}_{\bar{\tau}}^{1 \beta}}{d \tau}\right)^{2}+\frac{\partial^{2} \mathbf{g}_{\gamma \beta}}{\partial \mathbf{Y}_{\bar{\tau}}^{1}{ }^{\alpha 2}} \frac{d^{2} \mathbf{Y}_{\overline{\bar{\tau}}}^{{ }^{\alpha}}}{d \tau^{2}}+\frac{\partial^{3} \mathbf{g}_{\gamma \beta}}{\partial \mathbf{Y}_{\bar{\tau}}^{1^{\alpha 3}}}\left(\frac{d \mathbf{Y}_{\bar{\tau}}^{1^{\alpha}}}{d \tau}\right)^{2}\right) \\
& \left.\frac{d^{3} \mathbf{Y}_{\bar{\tau}}^{1^{\alpha}}}{d \tau^{3}} \frac{d \mathbf{Y}_{\bar{\tau}}^{1 \beta}}{d \tau}\right)
\end{aligned}
$$


Proof. In fact, the following relations hold:

$$
\begin{aligned}
& -\mathbf{D}^{1}\left(\frac{\partial L}{\partial \mathbf{D}^{1} \mathbf{Y}_{\bar{\tau}}^{1 \mu}}\right)=\mathbf{D}^{1}\left(\frac{1}{L_{1}} \mathbf{g}_{\alpha \gamma} \mathbf{D}^{1} \mathbf{Y}_{\bar{\tau}}^{1}{ }^{\alpha}\right)=\left(L_{1} \frac{d}{d \tau}-i \bar{\tau} L_{1}^{3} \frac{d^{3}}{d \tau^{3}}\right)\left(\mathbf{g}_{\alpha \gamma}\left(\frac{d \mathbf{Y}_{\bar{\tau}}^{1}}{d \tau}-i \bar{\tau} L_{1}^{2} \frac{d^{3} \mathbf{Y}_{\bar{\tau}}^{\alpha^{\alpha}}}{d \tau^{3}}\right)\right) \\
& \frac{\partial L_{1}}{\partial \mathbf{Y}_{\bar{\tau}}^{1 \gamma}}=-\frac{1}{2} L_{1} \frac{\partial \mathbf{g}_{\alpha \beta}}{\partial \mathbf{Y}_{\bar{\tau}}^{1 \gamma}}\left(\frac{d \mathbf{Y}_{\bar{\tau}}^{1}}{d \tau}-i \bar{\tau} L_{1}^{2} \frac{d^{3} \mathbf{Y}_{\bar{\tau}}^{1}}{d \tau^{3}}\right)\left(\frac{d \mathbf{Y}_{\bar{\tau}}^{1}{ }^{\beta}}{d \tau}-i \bar{\tau} L_{1}^{2} \frac{d^{3} \mathbf{Y}_{\overline{\bar{\tau}}}^{{ }^{\beta}}}{d \tau^{3}}\right) \\
& \frac{\partial L_{1}}{\partial \mathbf{D}^{1} Y_{\bar{\tau}}^{1 \gamma}}=-\frac{1}{2 L_{1}} \mathbf{g}_{\alpha \beta}\left(\delta_{\alpha \gamma} \mathbf{D}^{1} \mathbf{Y}_{\bar{\tau}}^{1 \beta}+\delta_{\beta \gamma} \mathbf{D}^{1} \mathbf{Y}_{\bar{\tau}}^{1 \alpha}\right)=-\frac{1}{L_{1}} \mathbf{g}_{\alpha \gamma} \mathbf{D}^{1} \mathbf{Y}_{\bar{\tau}}^{1 \alpha} \\
& =L_{1} \frac{d}{d \tau}\left(\mathbf{g}_{\alpha \gamma} \frac{d x^{\alpha}}{d \tau}\right)-i \bar{\tau} L_{1}^{3} \frac{d}{d \tau}\left(\mathbf{g}_{\alpha \gamma} \frac{d^{3} \mathbf{Y}_{\bar{\tau}}^{{ }^{\alpha}}}{d \tau^{3}}\right) \\
& \frac{d}{d \tau}\left(\mathbf{g}_{\alpha \gamma} \frac{d \mathbf{Y}_{\bar{\tau}}^{1}{ }^{\alpha}}{d \tau}\right)=\mathbf{g}_{\alpha \gamma} \frac{d^{2} \mathbf{Y}_{\bar{\tau}}^{1^{\alpha}}}{d \tau^{2}}+\frac{1}{2}\left(\frac{\partial \mathbf{g}_{\alpha \gamma}}{\partial \mathbf{Y}_{\bar{\tau}}^{1 \beta}}+\frac{\partial \mathbf{g}_{\gamma \beta}}{\partial \mathbf{Y}_{\bar{\tau}}^{1^{\alpha}}}\right) \frac{d \mathbf{Y}_{\bar{\tau}}^{1}}{d \tau} \frac{d \mathbf{Y}_{\bar{\tau}}^{1^{\beta}}}{d \tau} \\
& \frac{d}{d \tau}\left(\mathbf{g}_{\alpha \gamma} \frac{d^{3} \mathbf{Y}_{\bar{\tau}}^{1}}{d \tau^{3}}\right)=\mathbf{g}_{\alpha \gamma} \frac{d^{4} \mathbf{Y}_{\bar{\tau}}^{\alpha}}{d \tau^{4}}+\frac{1}{2}\left(\frac{\partial \mathbf{g}_{\alpha \gamma}}{\partial \mathbf{Y}_{\bar{\tau}}^{1^{\beta}}}+\frac{\partial \mathbf{g}_{\gamma \beta}}{\partial \mathbf{Y}_{\bar{\tau}}^{1^{\alpha}}}\right) \frac{d^{3} \mathbf{Y}_{\bar{\tau}}^{1^{\alpha}}}{d \tau^{3}} \frac{d \mathbf{Y}_{\bar{\tau}}^{1^{\beta}}}{d \tau}, \\
& \frac{d^{3}}{d \tau^{3}}\left(\mathbf{g}_{\alpha \gamma} \frac{d \mathbf{Y}_{\bar{\tau}}^{1}{ }^{\alpha}}{d \tau}\right)=\mathbf{g}_{\alpha \gamma} \frac{d^{4} \mathbf{Y}_{\bar{\tau}}^{{ }^{\alpha}}}{d \tau^{4}}+\frac{1}{2}\left(\frac{\partial \mathbf{g}_{\alpha \gamma}}{\partial \mathbf{Y}_{\bar{\tau}}^{1^{\beta}}}+\frac{\partial \mathbf{g}_{\gamma \beta}}{\partial \mathbf{Y}_{\bar{\tau}}^{1 \alpha}}\right) \frac{d \mathbf{Y}_{\bar{\tau}}^{1^{\beta}}}{d \tau} \frac{d^{3} \mathbf{Y}_{\bar{\tau}}^{{ }^{\alpha}}}{d \tau^{3}} \\
& -i \bar{\tau} L_{1}^{3} \frac{d^{3}}{d \tau^{3}}\left(\mathbf{g}_{\alpha \gamma} \frac{d \mathbf{Y}_{\bar{\tau}}^{1^{\alpha}}}{d \tau}\right)-\bar{\tau}^{2} L_{1}^{5} \frac{d^{3}}{d \tau^{3}}\left(\mathbf{g}_{\alpha \gamma} \frac{d^{3} \mathbf{Y}_{\bar{\tau}}^{{ }^{\alpha}}}{d \tau^{3}}\right) \\
& +\frac{1}{2}\left(\frac{\partial \mathbf{g}_{\alpha \gamma}}{\partial \mathbf{Y}_{\bar{\tau}}^{1^{\beta}}}+\frac{\partial \mathbf{g}_{\gamma \beta}}{\partial \mathbf{Y}_{\bar{\tau}}^{1}}\right)\left(\frac{d \mathbf{Y}_{\bar{\tau}}^{1}}{d \tau} \frac{d^{3} \mathbf{Y}_{\bar{\tau}}^{1^{\alpha}}}{d \tau^{3}}+2 \frac{d^{2} \mathbf{Y}_{\bar{\tau}}^{1^{\alpha}}}{d \tau^{2}} \frac{d^{2} \mathbf{Y}_{\bar{\tau}}^{{ }^{\beta}}}{d \tau^{2}}+\frac{d \mathbf{Y}_{\bar{\tau}}^{1^{\alpha}}}{d \tau} \frac{d^{3} \mathbf{Y}_{\bar{\tau}}^{{ }^{\beta}}}{d \tau^{3}}\right) \\
& +\frac{1}{2}\left(\frac{\partial^{2} \mathbf{g}_{\alpha \gamma}}{\partial \mathbf{Y}_{\bar{\tau}}^{1 \beta 2}} \frac{d \mathbf{Y}_{\bar{\tau}}^{1 \beta}}{d \tau}+\frac{\partial^{2} \mathbf{g}_{\gamma \beta} \beta}{\partial \mathbf{Y}_{\bar{\tau}}^{1}{ }^{\alpha 2}} \frac{d \mathbf{Y}_{\bar{\tau}}^{\alpha}}{d \tau}\right)\left(2 \frac{d^{2} \mathbf{Y}_{\bar{\tau}}^{1^{\alpha}}}{d \tau^{2}} \frac{d \mathbf{Y}_{\bar{\tau}}^{1 \beta}}{d \tau}+\frac{d \mathbf{Y}_{\bar{\tau}}^{\alpha}}{d \tau} \frac{d^{2} \mathbf{Y}_{\bar{\tau}}^{1^{\beta}}}{d \tau^{2}}\right) \\
& \frac{d^{3}}{d \tau^{3}}\left(\mathbf{g}_{\alpha \gamma} \frac{d^{3} \mathbf{Y}_{\bar{\tau}}^{1}{ }^{\alpha}}{d \tau^{3}}\right)=\mathbf{g}_{\alpha \gamma} \frac{d^{6} \mathbf{Y}_{\bar{\tau}}^{1^{\alpha}}}{d \tau^{6}}+\frac{1}{2}\left(\frac{\partial \mathbf{g}_{\alpha \gamma}}{\partial \mathbf{Y}_{\bar{\tau}}^{1^{\beta}}}+\frac{\partial \mathbf{g}_{\gamma \beta}}{\partial \mathbf{Y}_{\bar{\tau}}^{1^{\alpha}}}\right) \frac{d \mathbf{Y}_{\bar{\tau}}^{{ }^{\beta}}}{d \tau} \frac{d^{5} \mathbf{Y}_{\bar{\tau}}^{{ }^{\alpha}}}{d \tau^{5}}
\end{aligned}
$$




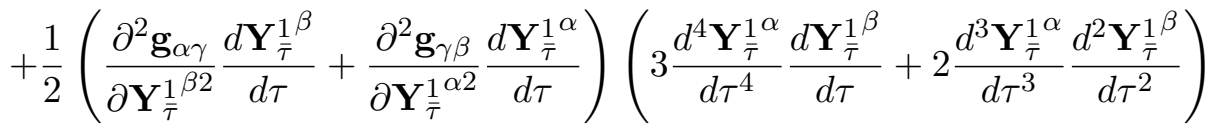

$$
\begin{aligned}
& +\frac{1}{2}\left(\frac{\partial \mathbf{g}_{\alpha \gamma}}{\partial \mathbf{Y}_{\bar{\tau}}^{1}{ }^{\beta}}+\frac{\partial \mathbf{g}_{\gamma \beta}}{\partial \mathbf{Y}_{\bar{\tau}}^{1}{ }^{\alpha}}\right)\left(2 \frac{d^{5} \mathbf{Y}_{\bar{\tau}}^{1}}{d \tau^{5}} \frac{d \mathbf{Y}_{\bar{\tau}}^{1}{ }^{\beta}}{d \tau}+3 \frac{d^{4} \mathbf{Y}_{\bar{\tau}}^{1^{\alpha}}}{d \tau^{4}} \frac{d^{2} \mathbf{Y}_{\bar{\tau}}^{1}{ }^{\beta}}{d \tau^{2}}+\frac{d^{3} \mathbf{Y}_{\bar{\tau}}^{1}}{d \tau^{3}} \frac{d^{3} \mathbf{Y}_{\overline{\bar{\tau}}}^{1^{\beta}}}{d \tau^{3}}\right) \\
& +\frac{1}{2}\left(\frac{\partial^{2} \mathbf{g}_{\alpha \gamma}}{\partial \mathbf{Y}_{\bar{\tau}}^{1 \beta 2}} \frac{d^{2} \mathbf{Y}_{\bar{\tau}}^{1^{\beta}}}{d \tau^{2}}+\frac{\partial^{3} \mathbf{g}_{\alpha \gamma}}{\partial \mathbf{Y}_{\bar{\tau}}^{1 \beta 3}}\left(\frac{d \mathbf{Y}_{\bar{\tau}}^{1 \beta}}{d \tau}\right)^{2}+\frac{\partial^{2} g_{\gamma \beta}}{\partial \mathbf{Y}_{\bar{\tau}}^{1}{ }^{\alpha 2}} \frac{d^{2} \mathbf{Y}_{\overline{\bar{\tau}}}^{1^{\alpha}}}{d \tau^{2}}+\frac{\partial^{3} g_{\gamma \beta}}{\partial \mathbf{Y}_{\bar{\tau}}^{1}{ }^{\alpha 3}}\left(\frac{d \mathbf{Y}_{\bar{\tau}}^{1^{\alpha}}}{d \tau}\right)^{2}\right) \\
& \frac{d^{3} \mathbf{Y}_{\bar{\tau}}^{1 \alpha}}{d \tau^{3}} \frac{d \mathbf{Y}_{\bar{\tau}}^{1 \beta}}{d \tau}
\end{aligned}
$$

Expanding terms and using relations (2.10), (2.11) and (2.12) we find equation (2.13).

Lemma 2.2. The Lagrangian is different from zero; then we can split equation (2.13) into three differential equations which are respectively factors of $L_{1}^{0}, L_{1}^{2}, L_{1}^{4}$ as follows:

$$
\begin{aligned}
& \mathbf{g}_{\alpha \gamma} \frac{d^{2} \mathbf{Y}_{\bar{\tau}}^{1^{\alpha}}}{d \tau^{2}}+\mathbf{g}_{\alpha \gamma} \Gamma_{\delta \beta}^{\alpha} \frac{d \mathbf{Y}_{\bar{\tau}}^{1 \delta}}{d \tau} \frac{d \mathbf{Y}_{\bar{\tau}}^{1 \beta}}{d \tau}=0 \\
& \mathbf{g}_{\alpha \gamma} \frac{d^{4} \mathbf{Y}_{\bar{\tau}}^{1^{\alpha}}}{d \tau^{4}}+\mathbf{g}_{\alpha \gamma} \Gamma_{\delta \beta}^{\alpha}\left(\frac{d^{3} \mathbf{Y}_{\bar{\tau}}^{1^{\delta}}}{d \tau^{3}} \frac{d \mathbf{Y}_{\bar{\tau}}^{1^{\beta}}}{d \tau}+\frac{d \mathbf{Y}_{\bar{\tau}}^{1^{\delta}}}{d \tau} \frac{d^{3} \mathbf{Y}_{\bar{\tau}}^{1^{\beta}}}{d \tau^{3}}\right) \\
& +\frac{1}{2}\left(\frac{\partial \mathbf{g}_{\alpha \gamma}}{\partial \mathbf{Y}_{\bar{\tau}}^{1 \beta}}+\frac{\partial \mathbf{g}_{\gamma \beta}}{\partial \mathbf{Y}_{\bar{\tau}}^{1^{\alpha}}}\right)\left(\frac{d \mathbf{Y}_{\bar{\tau}}^{1^{\beta}}}{d \tau} \frac{d^{3} \mathbf{Y}_{\bar{\tau}}^{\alpha}}{d \tau^{3}}+2 \frac{d^{2} \mathbf{Y}_{\bar{\tau}}^{1^{\alpha}}}{d \tau^{2}} \frac{d^{2} \mathbf{Y}_{\bar{\tau}}^{1^{\beta}}}{d \tau^{2}}\right) \\
& +\frac{1}{2}\left(2 \frac{d^{2} \mathbf{Y}_{\bar{\tau}}^{1}{ }^{\alpha}}{d \tau^{2}} \frac{d \mathbf{Y}_{\bar{\tau}}^{{ }^{\beta}}}{d \tau}+\frac{d \mathbf{Y}_{\bar{\tau}}^{1 \alpha}}{d \tau} \frac{d^{2} \mathbf{Y}_{\bar{\tau}}^{1 \beta}}{d \tau^{2}}\right)\left(\frac{\partial^{2} \mathbf{g}_{\alpha \gamma}}{\partial \mathbf{Y}_{\bar{\tau}}^{1 \beta 2}} \frac{d \mathbf{Y}_{\bar{\tau}}^{1 \beta}}{d \tau}+\frac{\partial^{2} \mathbf{g}_{\gamma \beta}}{\partial \mathbf{Y}_{\bar{\tau}}^{1^{\alpha 2}}} \frac{d \mathbf{Y}_{\bar{\tau}}^{1^{\alpha}}}{d \tau}\right)=0 \\
& \mathbf{g}_{\alpha \gamma} \frac{d^{6} \mathbf{Y}_{\overline{\bar{\tau}}}^{1^{\alpha}}}{d \tau^{6}}+\mathbf{g}_{\alpha \gamma} \Gamma_{\delta \beta}^{\alpha} \frac{d^{3} \mathbf{Y}_{\bar{\tau}}^{1}{ }^{\delta}}{d \tau^{3}} \frac{d^{3} \mathbf{Y}_{\bar{\tau}}^{1 \beta}}{d \tau^{3}} \\
& +\frac{1}{2}\left(\frac{\partial^{2} \mathbf{g}_{\alpha \gamma}}{\partial \mathbf{Y}_{\bar{\tau}}^{1 \beta 2}} \frac{d Y_{\bar{\tau}}^{1}}{d \tau}+\frac{\partial^{2} \mathbf{g}_{\gamma \beta}}{\partial \mathbf{Y}_{\bar{\tau}}^{1}{ }^{\alpha 2}} \frac{d \mathbf{Y}_{\bar{\tau}}^{1 \alpha}}{d \tau}\right)\left(3 \frac{d^{4} \mathbf{Y}_{\bar{\tau}}^{1}{ }^{\alpha}}{d \tau^{4}} \frac{d \mathbf{Y}_{\bar{\tau}}^{1 \beta}}{d \tau}+2 \frac{d^{3} \mathbf{Y}_{\bar{\tau}}^{1}{ }^{\alpha}}{d \tau^{3}} \frac{d^{2} \mathbf{Y}_{\bar{\tau}}^{1 \beta}}{d \tau^{2}}\right)
\end{aligned}
$$




$$
\begin{aligned}
& +\frac{1}{2}\left(\frac{\partial \mathbf{g}_{\alpha \gamma}}{\partial \mathbf{Y}_{\bar{\tau}}^{1 \beta}}+\frac{\partial \mathbf{g}_{\gamma \beta}}{\partial \mathbf{Y}_{\bar{\tau}}^{1}}\right)\left(2 \frac{d^{5} \mathbf{Y}_{\bar{\tau}}^{1}}{d \tau^{5}} \frac{d \mathbf{Y}_{\bar{\tau}}^{1 \beta}}{d \tau}+3 \frac{d^{4} \mathbf{Y}_{\bar{\tau}}^{1}{ }^{\alpha}}{d \tau^{4}} \frac{d^{2} \mathbf{Y}_{\bar{\tau}}^{1}{ }^{\beta}}{d \tau^{2}}+\frac{d^{3} \mathbf{Y}_{\bar{\tau}}^{1^{\alpha}}}{d \tau^{3}} \frac{d^{3} \mathbf{Y}_{\bar{\tau}}^{1 \beta}}{d \tau^{3}}\right) \\
& +\frac{1}{2}\left(\frac{\partial^{2} \mathbf{g}_{\alpha \gamma}}{\partial \mathbf{Y}_{\bar{\tau}}^{1}{ }^{\beta 2}} \frac{d^{2} \mathbf{Y}_{\bar{\tau}}^{{ }^{\beta}}}{d \tau^{2}}+\frac{\partial^{3} \mathbf{g}_{\alpha \gamma}}{\partial \mathbf{Y}_{\bar{\tau}}^{1 \beta 3}}\left(\frac{d \mathbf{Y}_{\bar{\tau}}^{1 \beta}}{d \tau}\right)^{2}+\frac{\partial^{2} \mathbf{g}_{\gamma \beta}}{\partial \mathbf{Y}_{\bar{\tau}}^{1^{\alpha 2}}} \frac{d^{2} \mathbf{Y}_{\bar{\tau}}^{{ }^{\alpha}}}{d \tau^{2}}+\frac{\partial^{3} \mathbf{g}_{\gamma \beta}}{\partial \mathbf{Y}_{\bar{\tau}}^{1}{ }^{\alpha 3}}\left(\frac{d \mathbf{Y}_{\bar{\tau}}^{{ }^{\alpha}}}{d \tau}\right)^{2}\right) \\
& \frac{d^{3} \mathbf{Y}_{\bar{\tau}}^{1 \alpha}}{d \tau^{3}} \frac{d \mathbf{Y}_{\bar{\tau}}^{1^{\beta}}}{d \tau}=0
\end{aligned}
$$

Here $\boldsymbol{\Gamma}_{\delta \beta}^{\mu}=\frac{1}{2} \mathbf{g}^{\mu \gamma}\left(\frac{\partial \mathbf{g}_{\gamma \delta}}{\partial \mathbf{Y}_{\bar{\tau}}^{1 \beta}}+\frac{\partial \mathbf{g}_{\gamma \beta}}{\partial \mathbf{Y}_{\bar{\tau}}^{1 \delta}}-\frac{\partial \mathbf{g}_{\delta \beta}}{\partial \mathbf{Y}_{\bar{\tau}}^{1 / \gamma}}\right)$ is the Christoffel symbol, $\mathbf{Y}_{\bar{\tau}}^{1 \beta 2}=$ $\left(\mathbf{Y}_{\bar{\tau}}^{1^{\beta}}\right)^{2}$ and so on.

The proof is direct. In what follows we will be interested mainly in equation (2.14) which already yields higher-order derivative terms.

Definition 2.4. We define the non-local-in-time complexified metric up to $1^{\text {st }}$-order in $\bar{\tau}$ by:

$$
\mathbf{g}_{\alpha \beta}\left(\mathbf{Y}_{\bar{\tau}}^{\sigma 1}\right) \equiv \mathbf{g}_{\alpha \beta}\left(\mathbf{X}^{\sigma}-i \bar{\tau} \dot{\mathbf{X}}^{\sigma}\right)=g_{\alpha \beta}-i \bar{\tau} \dot{\mathbf{X}}^{\sigma} \frac{\partial g_{\alpha \beta}}{\partial \mathbf{X}^{\sigma}}
$$

Lemma 2.3. The non-local-in-time complexified Christoffel symbol is:

$$
\Gamma_{\delta \beta}^{\mu}=\mathbf{R}_{\delta \beta}^{\mu}-i \bar{\tau} I_{\delta \beta}^{\mu}
$$

where

$$
\begin{gathered}
\mathbf{R}_{\delta \beta}^{\mu}=\frac{1}{2} g^{\mu \nu}\left(\frac{\partial g_{\gamma \delta}}{\partial \mathbf{X}^{\beta}}+\frac{\partial g_{\gamma \beta}}{\partial \mathbf{X}^{\delta}}-\frac{\partial g_{\delta \beta}}{\partial \mathbf{X}^{\gamma}}\right) \\
-\frac{1}{2} \bar{\tau}^{2} \dot{\mathbf{X}}^{\sigma} \frac{\partial g^{\mu \nu}}{\partial \mathbf{X}^{\sigma}}\left(\dot{\mathbf{X}}^{\beta} \frac{\partial^{2} g_{\gamma \delta}}{\partial \mathbf{X}^{\beta 2}}+\dot{\mathbf{X}}^{\delta} \frac{\partial^{2} g_{\gamma \beta}}{\partial \mathbf{X}^{\delta 2}}+\dot{\mathbf{X}}^{\gamma} \frac{\partial^{2} g_{\delta \beta}}{\partial \mathbf{X}^{\gamma 2}}\right)
\end{gathered}
$$

and

$$
\begin{aligned}
\mathbf{I}_{\delta \beta}^{\mu}= & \frac{1}{2} g^{\mu \nu}\left(\dot{\mathbf{X}}^{\beta} \frac{\partial^{2} g_{\gamma \delta}}{\partial \mathbf{X}^{\beta 2}}+\dot{\mathbf{X}}^{\delta} \frac{\partial^{2} g_{\gamma \beta}}{\partial \mathbf{X}^{\delta 2}}+\dot{\mathbf{X}}^{\gamma} \frac{\partial^{2} g_{\delta \beta}}{\partial \mathbf{X}^{\gamma 2}}\right) \\
& +\frac{1}{2} \dot{\mathbf{X}}^{\sigma} \frac{\partial g^{\mu \nu}}{\partial \mathbf{X}^{\sigma}}\left(\frac{\partial g_{\gamma \delta}}{\partial \mathbf{X}^{\beta}}+\frac{\partial g_{\gamma \beta}}{\partial \mathbf{X}^{\delta}}-\frac{\partial g_{\delta \beta}}{\partial \mathbf{X}^{\gamma}}\right)
\end{aligned}
$$


Proof. In fact, using equation (2.17), we can write:

$$
\begin{gathered}
\frac{\partial \mathbf{g}_{\gamma \delta}}{\partial \mathbf{Y}_{\bar{\tau}}^{1 \beta}}=\frac{\partial}{\partial \mathbf{Y}_{\bar{\tau}}^{1^{\beta}}}\left(g_{\gamma \delta}-i \bar{\tau} \dot{\mathbf{X}}^{\beta} \frac{\partial g_{\gamma \delta}}{\partial \mathbf{X}^{\beta}}\right), \\
=\frac{\partial g_{\gamma \delta}}{\partial \mathbf{Y}_{\bar{\tau}}^{1^{\beta}}}-i \bar{\tau} \frac{\partial}{\partial \mathbf{Y}_{\bar{\tau}}^{{ }^{\beta}}}\left(\dot{\mathbf{X}}^{\beta} \frac{\partial g_{\gamma \delta}}{\partial \mathbf{X}^{\beta}}\right)=\frac{\partial g_{\gamma \delta}}{\partial \mathbf{X}^{\beta}}-i \bar{\tau} \dot{\mathbf{X}}^{\beta} \frac{\partial^{2} g_{\gamma \delta}}{\partial \mathbf{X}^{\beta 2}} .
\end{gathered}
$$

The same results holds for the rest of terms in $\Gamma_{\delta \beta}^{\mu}$ and consequently we can write:

$$
\begin{gathered}
\Gamma_{\delta \beta}^{\mu}=\frac{1}{2}\left(g^{\mu \nu}-i \bar{\tau} \dot{\mathbf{X}}^{\sigma} \frac{\partial g^{\mu \nu}}{\partial \mathbf{X}^{\sigma}}\right)\left(\frac{\partial g_{\gamma \delta}}{\partial \mathbf{X}^{\beta}}+\frac{\partial g_{\gamma \beta}}{\partial \mathbf{X}^{\delta}}-\frac{\partial g_{\delta \beta}}{\partial \mathbf{X}^{\gamma}}\right. \\
\left.-i \bar{\tau}\left(\dot{\mathbf{X}}^{\beta} \frac{\partial^{2} g_{\gamma \delta}}{\partial \mathbf{X}^{\beta 2}}+\dot{\mathbf{X}}^{\delta} \frac{\partial^{2} g_{\gamma \beta}}{\partial \mathbf{X}^{\delta 2}}+\dot{X}^{\gamma} \frac{\partial^{2} g_{\delta \beta}}{\partial \mathbf{X}^{\gamma 2}}\right)\right) \\
=\left(\frac{1}{2} g^{\mu \nu}\left(\frac{\partial g_{\gamma \delta}}{\partial \mathbf{X}^{\beta}}+\frac{\partial g_{\gamma \beta}}{\partial \mathbf{X}^{\delta}}-\frac{\partial g_{\delta \beta}}{\partial \mathbf{X}^{\gamma}}\right)-\frac{1}{2} \bar{\tau}^{2} \dot{\mathbf{X}}^{\sigma} \frac{\partial g^{\mu \nu}}{\partial \mathbf{X}^{\sigma}}\left(\dot{\mathbf{X}}^{\beta} \frac{\partial^{2} g_{\gamma \delta}}{\partial \mathbf{X}^{\beta 2}}+\dot{\mathbf{X}}^{\delta} \frac{\partial^{2} g_{\gamma \beta}}{\partial \mathbf{X}^{\delta 2}}+\dot{\mathbf{X}}^{\gamma} \frac{\partial^{2} g_{\delta \beta}}{\partial \mathbf{X}^{\gamma 2}}\right)\right) \\
-i \bar{\tau}\left(\frac{1}{2} g^{\mu \nu}\left(\dot{X}^{\beta} \frac{\partial^{2} g_{\gamma \delta}}{\partial X^{\beta 2}}+\dot{X}^{\delta} \frac{\partial^{2} g_{\gamma \beta}}{\partial X^{\delta 2}}+\dot{X}^{\gamma} \frac{\partial^{2} g_{\delta \beta}}{\partial X^{\gamma 2}}\right)+\right. \\
\left.\frac{1}{2} \dot{X}^{\sigma} \frac{\partial g^{\mu \nu}}{\partial X^{\sigma}}\left(\frac{\partial g_{\gamma \delta}}{\partial X^{\beta}}+\frac{\partial g_{\gamma \beta}}{\partial X^{\delta}}-\frac{\partial g_{\delta \beta}}{\partial X^{\gamma}}\right)\right), \\
\equiv R_{\delta \beta}^{\mu}-i \bar{\tau} I_{\delta \beta}^{\mu} .
\end{gathered}
$$

Remark 2.1. It is obvious that when $\bar{\tau}=0$ equation (2.18) is reduced to the standard Christoffel symbol.

Corollary 2.1. The non-local-in-time complexified geodesic equation (2.14) is splitted into two parts:

$$
\begin{aligned}
& \frac{d^{2} \mathbf{X}^{\mu}}{d \tau^{2}}+\mathbf{R}_{\delta \beta}^{\mu}\left(\frac{d \mathbf{X}^{\delta}}{d \tau} \frac{d \mathbf{X}^{\beta}}{d \tau}-\bar{\tau}^{2} \frac{d^{2} \mathbf{X}^{\delta}}{d \tau^{2}} \frac{d^{2} \mathbf{X}^{\beta}}{d \tau^{2}}\right)-\bar{\tau}^{2} \mathbf{I}_{\delta \beta}^{\mu}\left(\frac{d^{2} \mathbf{X}^{\delta}}{d \tau^{2}} \frac{d \mathbf{X}^{\beta}}{d \tau}+\frac{d \mathbf{X}^{\delta}}{d \tau} \frac{d^{2} \mathbf{X}^{\beta}}{d \tau^{2}}\right)=0 \\
& \frac{d^{3} \mathbf{X}^{\mu}}{d \tau^{3}}+\mathbf{R}_{\delta \beta}^{\mu}\left(\frac{d^{2} \mathbf{X}^{\delta}}{d \tau^{2}} \frac{d \mathbf{X}^{\beta}}{d \tau}+\frac{d \mathbf{X}^{\delta}}{d \tau} \frac{d^{2} \mathbf{X}^{\beta}}{d \tau^{2}}\right)+\mathbf{I}_{\delta \beta}^{\mu}\left(\frac{d \mathbf{X}^{\delta}}{d \tau} \frac{d \mathbf{X}^{\beta}}{d \tau}-\bar{\tau}^{2} \frac{d^{2} \mathbf{X}^{\delta}}{d \tau^{2}} \frac{d^{2} \mathbf{X}^{\beta}}{d \tau^{2}}\right)=0 .
\end{aligned}
$$


Proof. Using $\mathbf{Y}_{\tau}^{1}=\mathbf{X}-i \bar{\tau} \dot{\mathbf{X}}$ and equation (2.18), we can split equation (2.14) into real and complexified parts.

It is obvious that equation $(2.22)$ is a $3^{\text {rd }}$-order differential equation in contrast to the standard geodesic equation which is a $2^{\text {nd }}$-order differential equation. By neglecting terms on $\bar{\tau}^{2}$, equations (2.21) and (2.22) are reduced respectively to:

$$
\frac{d^{2} \mathbf{X}^{\mu}}{d \tau^{2}}+\mathbf{R}_{\delta \beta}^{\mu} \frac{d \mathbf{X}^{\delta}}{d \tau} \frac{d \mathbf{X}^{\beta}}{d \tau}=0
$$

and

$$
\frac{d^{3} \mathbf{X}^{\mu}}{d \tau^{3}}+\mathbf{R}_{\delta \beta}^{\mu}\left(\frac{d^{2} \mathbf{X}^{\delta}}{d \tau^{2}} \frac{d \mathbf{X}^{\beta}}{d \tau}+\frac{d \mathbf{X}^{\delta}}{d \tau} \frac{d^{2} \mathbf{X}^{\beta}}{d \tau^{2}}\right)+\mathbf{I}_{\delta \beta}^{\mu} \frac{d \mathbf{X}^{\delta}}{d \tau} \frac{d \mathbf{X}^{\beta}}{d \tau}=0
$$

By differential equation (2.23) with respect to $\tau$, we find:

$$
\frac{d^{3} \mathbf{X}^{\mu}}{d \tau^{3}}+\mathbf{R}_{\delta \beta}^{\mu}\left(\frac{d^{2} \mathbf{X}^{\delta}}{d \tau^{2}} \frac{d \mathbf{X}^{\beta}}{d \tau}+\frac{d \mathbf{X}^{\delta}}{d \tau} \frac{d^{2} \mathbf{X}^{\beta}}{d \tau^{2}}\right)+\frac{d \mathbf{R}_{\delta \beta}^{\mu}}{d \tau} \frac{d \mathbf{X}^{\delta}}{d \tau} \frac{d \mathbf{X}^{\beta}}{d \tau}=0
$$

and after comparing with equation (2.24) we find:

$$
\frac{d \mathbf{R}_{\delta \beta}^{\mu}}{d \tau}=\mathbf{I}_{\delta \beta}^{\mu} .
$$

Corollary 2.2. The spacetime metric $d \mathbf{s}^{2}=\mathbf{g}_{\alpha \beta} d \mathbf{Y}_{\bar{\tau}}^{1^{\alpha}} d \mathbf{Y}_{\bar{\tau}}^{1^{\beta}}$ is complexified and is splitted as follows:

$$
d \mathbf{s}^{2}=\mathbf{g}_{\alpha \beta} d \mathbf{Y}_{\bar{\tau}}^{1^{\alpha}} d \mathbf{Y}_{\bar{\tau}}^{1^{\beta}}=d \mathbf{s}_{\mathbf{R}}{ }^{2}-i \bar{\tau} d \mathbf{s}_{\mathbf{I}}^{2}
$$

where

$$
d \mathbf{s}_{\mathbf{R}}^{2}=g_{\alpha \beta}\left(d \mathbf{X}^{\alpha} d \mathbf{X}^{\beta}-\bar{\tau}^{2} d \dot{\mathbf{X}}^{\alpha} d \dot{\mathbf{X}}^{\beta}\right)-\bar{\tau}^{2} \dot{\mathbf{X}}^{\sigma} \frac{\partial g_{\alpha \beta}}{\partial \mathbf{X}^{\sigma}}\left(d \mathbf{X}^{\alpha} d \dot{\mathbf{X}}^{\beta}+d \dot{\mathbf{X}}^{\alpha} d \mathbf{X}^{\beta}\right)
$$

and

$$
d \mathbf{s}_{\mathbf{I}}^{2}=g_{\alpha \beta}\left(d \mathbf{X}^{\alpha} d \dot{\mathbf{X}}^{\beta}+d \dot{\mathbf{X}}^{\alpha} d X^{\beta}\right)+\dot{\mathbf{X}}^{\sigma} \frac{\partial g_{\alpha \beta}}{\partial \mathbf{X}^{\sigma}}\left(d \mathbf{X}^{\alpha} d \mathbf{X}^{\beta}-\bar{\tau}^{2} d \dot{\mathbf{X}}^{\alpha} d \dot{\mathbf{X}}^{\beta}\right)
$$

By neglecting terms on $\bar{\tau}^{2}$, equations (2.28) and (2.29) are reduced respectively to: 


$$
d \mathbf{s}_{\mathbf{R}}^{2}=g_{\alpha \beta} d \mathbf{X}^{\alpha} d \mathbf{X}^{\beta}
$$

and

$$
d \mathbf{s}_{\mathbf{I}}^{2}=g_{\alpha \beta}\left(d \mathbf{X}^{\alpha} d \dot{\mathbf{X}}^{\beta}+d \dot{\mathbf{X}}^{\alpha} d \mathbf{X}^{\beta}\right)+\dot{\mathbf{X}}^{\sigma} \frac{\partial g_{\alpha \beta}}{\partial \mathbf{X}^{\sigma}} d \mathbf{X}^{\alpha} d \mathbf{X}^{\beta}
$$

Both equations are related by derivative with respect to $\tau$. It should be stressed that the complexified spacetime is explored in literature through different contexts and mainly in theoretical physics [?2,?4,?10,?27], differential geometry [?12,?29] and twistor theory [?22,?23,?31].

\section{Illustrations}

3.1: As a first illustration, we attack a classical gravitational problem. It is well-known that general relativity describes gravitation in terms of the spacetime curvature and without gravitation the spacetime possesses the Minkowski metric of special relativity $\eta_{\mu \nu}$. In our approach, the Minkowski metric is complexified and we define it as $\eta_{\mu \nu}$. It is worth-mentioning that the complexification of the Minkowski spacetime was discussed through different frameworks in literature [?6, ?21]. In weak spacetime curvature, the complexified metric is of the form $\mathbf{g}_{\mu \nu}=\eta_{\mu \nu}+\mathbf{h}_{\mu \nu}$ with $\left|h_{\mu \nu}\right|<<1$. For $n=1$ and up to the first order on $\bar{\tau}$ we can write:

$$
\mathbf{g}_{\mu \nu}=\eta_{\mu \nu}+h_{\mu \nu}-i \bar{\tau} \dot{\mathbf{X}}^{\sigma} \frac{\partial h_{\mu \nu}}{\partial \mathbf{X}^{\sigma}}
$$

where

$$
\mathbf{h}_{\mu \nu}=h_{\mu \nu}-i \bar{\tau} \dot{\mathbf{X}}^{\sigma} \frac{\partial h_{\mu \nu}}{\partial \mathbf{X}^{\sigma}}
$$

and

$$
\boldsymbol{\eta}_{\mu \nu}=\eta_{\mu \nu}-i \bar{\tau} \dot{\mathbf{X}}^{\sigma} \frac{\partial \eta_{\mu \nu}}{\partial \mathbf{X}^{\sigma}}=\eta_{\mu \nu}
$$

For the case of stationary metric, equations (2.19) and (2.20) give:

$$
\begin{gathered}
\mathbf{R}_{00}^{0}=\mathbf{I}_{00}^{0}=0, \\
\mathbf{R}_{00}^{i}=-\frac{1}{2} \eta^{i j} \frac{\partial h_{00}}{\partial \mathbf{X}^{j}},
\end{gathered}
$$




$$
\mathbf{I}_{00}^{i}=\frac{1}{2} \eta^{i j} \dot{\mathbf{X}}^{i} \frac{\partial^{2} h_{00}}{\partial \mathbf{X}^{i 2}}
$$

By considering a free-falling particle falling slowly in the stationary metric, equations (2.23) and (2.24) are reduced respectively to:

$$
\frac{d^{2} \mathbf{X}^{\mu}}{d \tau^{2}}+\mathbf{R}_{00}^{\mu}\left(\frac{d t}{d \tau}\right)^{2}=0
$$

and

$$
\frac{d^{3} \mathbf{X}^{\mu}}{d \tau^{3}}+2 \mathbf{R}_{00}^{\mu}\left(\frac{d^{2} t}{d \tau^{2}} \frac{d t}{d \tau}\right)+\mathbf{I}_{00}^{\mu}\left(\frac{d t}{d \tau}\right)^{2}=0
$$

where we have used the fact that $\mathbf{X}^{0}=t$ in units $c=G=1$ ( $c$ being the celerity of light and $G$ is the gravitational constant). Using equations (3.4)(3.6), we find: $d t / d \tau=k \in$ and besides equations (3.7) and (3.8) are reduced to:

$$
\frac{d^{2} \overrightarrow{\mathbf{X}}}{d t^{2}}=-\frac{1}{2} \nabla h_{00}
$$

and

$$
\frac{d^{3} \overrightarrow{\mathbf{X}}}{d t^{3}}=\frac{1}{2} \frac{d \overrightarrow{\mathbf{X}}}{d t} \Delta h_{00}
$$

Comparing equation (3.9) with the Newtonian equation describing the motion of a particle in a gravitational field $\boldsymbol{\Phi}$, i.e. $d^{2} \overrightarrow{\mathbf{X}} / d t^{2}=-\nabla \boldsymbol{\Phi}$, we observe that $h_{00}=2 \Phi$. After replacing into equation (3.10), we obtain:

$$
\frac{d^{3} \overrightarrow{\mathbf{X}}}{d t^{3}}=\frac{d \overrightarrow{\mathbf{X}}}{d t} \Delta \Phi
$$

This equation shows that the jerk $\overrightarrow{\mathcal{J}} \equiv d^{3} \overrightarrow{\mathbf{X}} / d t^{3}$ which is the rate of change of acceleration is related to the velocity $\overrightarrow{\mathcal{V}} \equiv d \overrightarrow{\mathbf{X}} / d t$ and the gravitational field by: $\vec{\jmath} \equiv \overrightarrow{\mathcal{V}} \Delta \boldsymbol{\Phi}$. This relation does not appear in the standard formalism. However, it is well-known from potential theory that $\Delta \boldsymbol{\Phi}=4 \pi G \rho$ (Poisson equation) where $\rho$ is the mass density and $G$ is the gravitational constant. Therefore we find: $\vec{\jmath}=4 \pi G \rho \overrightarrow{\mathcal{V}}$. However, for the case of a time-independent gravitational field, one can check after differentiating equation $d^{2} \overrightarrow{\mathbf{X}} / d t^{2}=$ $-\nabla \Phi$ with respect to time that the jerk is zero and therefore the Poisson equation is reduced to the Laplace equation $\Delta \boldsymbol{\Phi}=0$. Accordingly, in our 
approach, $g_{00}=1+h_{00}=1+2 \boldsymbol{\Phi}$ and the spacetime interval is $d s^{2}=-(1+$ $2 \boldsymbol{\Phi}) d t^{2}+d x^{2}+d y^{2}+d z^{2}$ where $\boldsymbol{\Phi}$ obeys equation the Laplace equation $\Delta \boldsymbol{\Phi}=0$. The solution of this equation gives $\boldsymbol{\Phi}=A-M / r, A$ is a free real constant. This solution describes in fact that the gravitational field of a mass $M$ at the origin of the coordinate. Therefore $g_{00}=1+h_{00}=1+2 A-2 M / r$. By setting $A=0$, we find $g_{00}=1-2 M / r$. This is interesting since we can obtain the Schwarzschild solution directly without using boundary conditions [?14].

3.2: As a second application, we would like to derive the geodesic deviation equation again for $n=1$ and up to the first order on $\bar{\tau}$. In fact, the geodesic deviation equation plays an important role in theoretical physics, gravitational theories and technical astrophysics and it is a natural extension from the geodesic equation [?11,?26]. In differential geometry, this equation is commonly recognized as the Hamilton-Jacobi equation whereas in general relativity this equation describes the dynamics of closed objects controlled by a spatially varying gravitational field. By considering a nearby point $\overline{\mathbf{X}}^{\mu}=\mathbf{X}^{\mu}+\boldsymbol{\xi}^{\mu}, \xi^{\mu}<<1$ and using the expansion of the Christoffel symbol up to the $1^{\text {st }}$-order [?18]:

$$
\begin{gathered}
\overline{\mathbf{R}}_{\delta \beta}^{\mu}\left(\overline{\mathbf{X}}^{\mu}\right)=\overline{\mathbf{R}}_{\delta \beta}^{\mu}\left(\mathbf{X}^{\mu}+\boldsymbol{\xi}^{\mu}\right)=\mathbf{R}_{\delta \beta}^{\mu}\left(\mathbf{X}^{\mu}\right)+\mathbf{R}_{\delta \beta, \lambda}^{\mu} \boldsymbol{\xi}^{\lambda}+\mathrm{O}\left(\boldsymbol{\xi}^{2 \lambda}\right), \\
\overline{\mathbf{I}}_{\delta \beta}^{\mu}\left(\overline{\mathbf{X}}^{\mu}\right)=\overline{\mathbf{I}}_{\delta \beta}^{\mu}\left(\mathbf{X}^{\mu}+\boldsymbol{\xi}^{\mu}\right)=\mathbf{I}_{\delta \beta}^{\mu}\left(\mathbf{X}^{\mu}\right)+\mathbf{I}_{\delta \beta, \lambda}^{\mu} \boldsymbol{\xi}^{\lambda}+\mathrm{O}\left(\boldsymbol{\xi}^{2 \lambda}\right),
\end{gathered}
$$

where $\mathbf{R}_{\delta \beta, \lambda}^{\mu}=\partial \mathbf{R}_{\delta \beta}^{\mu} / \partial x^{\lambda}$ and $\mathbf{I}_{\delta \beta, \lambda}^{\mu}=\partial \mathbf{I}_{\delta \beta}^{\mu} / \partial x^{\lambda}$, we can approximate equations (2.23) and (2.24) respectively by:

$$
\frac{d^{2} \boldsymbol{\xi}^{\mu}}{d \tau^{2}}+\frac{d^{2} \mathbf{X}^{\mu}}{d \tau^{2}}+\mathbf{R}_{\delta \beta}^{\mu} \frac{d \mathbf{X}^{\delta}}{d \tau} \frac{d \mathbf{X}^{\beta}}{d \tau}+2 \mathbf{R}_{\delta \beta}^{\mu} \frac{d \mathbf{X}^{\delta}}{d \tau} \frac{d \boldsymbol{\xi}^{\beta}}{d \tau}+\mathbf{R}_{\delta \beta, \lambda}^{\mu} \boldsymbol{\xi}^{\lambda} \frac{d \mathbf{X}^{\delta}}{d \tau} \frac{d \mathbf{X}^{\beta}}{d \tau}=0
$$

and

$$
\begin{array}{r}
\frac{d^{3} \mathbf{X}^{\mu}}{d \tau^{3}}+\frac{d^{3} \boldsymbol{\xi}^{\mu}}{d \tau^{3}}+\mathbf{R}_{\delta \beta}^{\mu}\left(\frac{d^{2} \mathbf{X}^{\delta}}{d \tau^{2}} \frac{d \mathbf{X}^{\beta}}{d \tau}+\frac{d \mathbf{X}^{\delta}}{d \tau} \frac{d^{2} \mathbf{X}^{\beta}}{d \tau^{2}}\right) \\
+2 \mathbf{R}_{\delta \beta}^{\mu} \frac{d^{2} \mathbf{X}^{\delta}}{d \tau^{2}} \frac{d \boldsymbol{\xi}^{\beta}}{d \tau}+\mathbf{R}_{\delta \beta, \lambda}^{\mu} \boldsymbol{\xi}^{\lambda} \frac{d \mathbf{X}^{\delta}}{d \tau} \frac{d^{2} \mathbf{X}^{\beta}}{d \tau^{2}} \\
+\mathbf{I}_{\delta \beta}^{\mu} \frac{d \mathbf{X}^{\delta}}{d \tau} \frac{d \mathbf{X}^{\beta}}{d \tau}+2 \mathbf{I}_{\delta \beta}^{\mu} \frac{d \mathbf{X}^{\delta}}{d \tau} \frac{d \boldsymbol{\xi}^{\beta}}{d \tau}+\mathbf{I}_{\delta \beta, \lambda}^{\mu} \boldsymbol{\xi}^{\lambda} \frac{d \mathbf{X}^{\delta}}{d \tau} \frac{d \mathbf{X}^{\beta}}{d \tau}=0 .
\end{array}
$$

Using equations (2.23) and (2.24), we can reduce equations (3.14) and (3.15) respectively to: 
Vol. LIV (2016) Higher-order geodesic equations ...

$$
\frac{d^{2} \boldsymbol{\xi}^{\mu}}{d \tau^{2}}+2 R_{\delta \beta}^{\mu} \frac{d \mathbf{X}^{\delta}}{d \tau} \frac{d \boldsymbol{\xi}^{\beta}}{d \tau}+R_{\delta \beta, \lambda}^{\mu} \boldsymbol{\xi}^{\lambda} \frac{d \mathbf{X}^{\delta}}{d \tau} \frac{d \mathbf{X}^{\beta}}{d \tau}=0
$$

and

$$
\begin{gathered}
\frac{d^{3} \boldsymbol{\xi}^{\mu}}{d \tau^{3}}+2 \mathbf{R}_{\delta \beta}^{\mu} \frac{d^{2} \mathbf{X}^{\delta}}{d \tau^{2}} \frac{d \boldsymbol{\xi}^{\beta}}{d \tau}+\mathbf{R}_{\delta \beta, \lambda}^{\mu} \boldsymbol{\xi}^{\lambda} \frac{d \mathbf{X}^{\delta}}{d \tau} \frac{d^{2} \mathbf{X}^{\beta}}{d \tau^{2}} \\
+2 \mathbf{I}_{\delta \beta}^{\mu} \frac{d \mathbf{X}^{\delta}}{d \tau} \frac{d \boldsymbol{\xi}^{\beta}}{d \tau}+\mathbf{I}_{\delta \beta, \lambda}^{\mu} \boldsymbol{\xi}^{\lambda} \frac{d \mathbf{X}^{\delta}}{d \tau} \frac{d \mathbf{X}^{\beta}}{d \tau}=0
\end{gathered}
$$

It may be checked that some mathematical algebra, equation (3.16) is reduced to the well-known geodesic deviation equation [18]:

$$
\frac{\hat{\mathcal{D}}^{2} \boldsymbol{\xi}^{\mu}}{\hat{\mathfrak{D}} \tau^{2}}+\mathbf{R}_{\delta \lambda \beta}^{\mu} \frac{d x^{\delta}}{d \tau} \boldsymbol{\xi}^{\lambda} \frac{d x^{\beta}}{d \tau}=0 .
$$

$\mathbf{R}_{\delta \lambda \beta}^{\alpha}=\Gamma_{\delta \beta, \lambda}^{\alpha}-\Gamma_{\delta \lambda, \beta}^{\alpha}+\Gamma_{\sigma \lambda}^{\alpha} \Gamma_{\delta \beta}^{\sigma}-\Gamma_{\sigma \beta}^{\alpha} \Gamma_{\delta \lambda}^{\sigma}$ is the curvature Riemann tensor and $\hat{\mathcal{D}} / \hat{\mathcal{D}} \tau$ is the covariant derivative which help us write equation (2.24) as $\hat{\mathcal{D}}^{2} \mathbf{X}^{\mu} / \hat{\mathcal{D}} \tau^{2}=0$. In return to equation (3.17), we can write first it in the form:

$$
\begin{gathered}
\frac{d^{3} \boldsymbol{\xi}^{\mu}}{d \tau^{3}}+2 \mathbf{R}_{\delta \beta}^{\mu} \frac{d^{2} \mathbf{X}^{\delta}}{d \tau^{2}} \frac{d \boldsymbol{\xi}^{\beta}}{d \tau}+2 \mathbf{I}_{\delta \beta}^{\mu} \frac{d \mathbf{X}^{\delta}}{d \tau} \frac{d \boldsymbol{\xi}^{\beta}}{d \tau} \\
=-\mathbf{R}_{\delta \beta, \lambda}^{\mu} \boldsymbol{\xi}^{\lambda} \frac{d X^{\delta}}{d \tau} \frac{d^{2} \mathbf{X}^{\beta}}{d \tau^{2}}-\mathbf{I}_{\delta \beta, \lambda}^{\mu} \boldsymbol{\xi}^{\lambda} \frac{d \mathbf{X}^{\delta}}{d \tau} \frac{d \mathbf{X}^{\beta}}{d \tau} .
\end{gathered}
$$

By adding to both sides of equation (3.19) the terms:

$$
\begin{gathered}
\mathbf{R}_{\delta \lambda, \beta}^{\mu} \boldsymbol{\xi}^{\lambda} \frac{d \mathbf{X}^{\delta}}{d \tau} \frac{d^{2} \mathbf{X}^{\beta}}{d \tau^{2}}+\mathbf{R}_{\sigma \beta}^{\mu} R_{\alpha \lambda}^{\sigma} \boldsymbol{\xi}^{\lambda} \frac{d \mathbf{X}^{\delta}}{d \tau} \frac{d^{2} \mathbf{X}^{\beta}}{d \tau^{2}}-\mathbf{R}_{\sigma \lambda}^{\mu} \mathbf{R}_{\delta \beta}^{\sigma} \boldsymbol{\xi}^{\lambda} \frac{d \mathbf{X}^{\delta}}{d \tau} \frac{d^{2} \mathbf{X}^{\beta}}{d \tau^{2}} \\
+I_{\delta \lambda, \beta}^{\mu} \boldsymbol{\xi}^{\lambda} \frac{d \mathbf{X}^{\delta}}{d \tau} \frac{d^{2} \mathbf{X}^{\beta}}{d \tau^{2}}+\mathbf{I}_{\sigma \beta}^{\mu} \mathbf{I}_{\alpha \lambda}^{\sigma} \boldsymbol{\xi}^{\lambda} \frac{d \mathbf{X}^{\delta}}{d \tau} \frac{d^{2} \mathbf{X}^{\beta}}{d \tau^{2}}-\mathbf{I}_{\sigma \lambda}^{\mu} \mathbf{I}_{\delta \beta}^{\sigma} \boldsymbol{\xi}^{\lambda} \frac{d \mathbf{X}^{\delta}}{d \tau} \frac{d^{2} \mathbf{X}^{\beta}}{d \tau^{2}}
\end{gathered}
$$

we find:

$$
\begin{gathered}
\frac{d^{3} \boldsymbol{\xi}^{\mu}}{d \tau^{3}}+2 \mathbf{R}_{\delta \beta}^{\mu} \frac{d^{2} \mathbf{X}^{\delta}}{d \tau^{2}} \frac{d \boldsymbol{\xi}^{\beta}}{d \tau}+\mathbf{R}_{\delta \lambda, \beta}^{\mu} \boldsymbol{\xi}^{\lambda} \frac{d \mathbf{X}^{\delta}}{d \tau} \frac{d^{2} \mathbf{X}^{\beta}}{d \tau^{2}} \\
+\mathbf{R}_{\sigma \beta}^{\mu} \mathbf{R}_{\alpha \lambda}^{\sigma} \boldsymbol{\xi}^{\lambda} \frac{d \mathbf{X}^{\delta}}{d \tau} \frac{d^{2} \mathbf{X}^{\beta}}{d \tau^{2}}-\mathbf{R}_{\sigma \lambda}^{\mu} \mathbf{R}_{\delta \beta}^{\sigma} \boldsymbol{\xi}^{\lambda} \frac{d \mathbf{X}^{\delta}}{d \tau} \frac{d^{2} \mathbf{X}^{\beta}}{d \tau^{2}}
\end{gathered}
$$




$$
\begin{aligned}
+2 \mathbf{I}_{\delta \beta}^{\mu} \frac{d \mathbf{X}^{\delta}}{d \tau} \frac{d \boldsymbol{\xi}^{\beta}}{d \tau} & +\mathbf{I}_{\delta \lambda, \beta}^{\mu} \boldsymbol{\xi}^{\lambda} \frac{d \mathbf{X}^{\delta}}{d \tau} \frac{d^{2} \mathbf{X}^{\beta}}{d \tau^{2}}+\mathbf{I}_{\sigma \beta}^{\mu} \mathbf{I}_{\alpha \lambda}^{\sigma} \boldsymbol{\xi}^{\lambda} \frac{d \mathbf{X}^{\delta}}{d \tau} \frac{d^{2} \mathbf{X}^{\beta}}{d \tau^{2}}-\mathbf{I}_{\sigma \lambda}^{\mu} \mathbf{I}_{\delta \beta}^{\sigma} \xi^{\lambda} \frac{d \mathbf{X}^{\delta}}{d \tau} \frac{d^{2} \mathbf{X}^{\beta}}{d \tau^{2}} \\
= & \left(\mathbf{R}_{\delta \lambda, \beta}^{\mu}-\mathbf{R}_{\delta \beta, \lambda}^{\mu}+\mathbf{R}_{\sigma \beta}^{\mu} \mathbf{R}_{\alpha \lambda}^{\sigma}-\mathbf{R}_{\sigma \lambda}^{\mu} \mathbf{R}_{\delta \beta}^{\sigma}\right) \boldsymbol{\xi}^{\lambda} \frac{d \mathbf{X}^{\delta}}{d \tau} \frac{d^{2} \mathbf{X}^{\beta}}{d \tau^{2}} \\
& +\left(\mathbf{I}_{\delta \lambda, \beta}^{\mu}-\mathbf{I}_{\delta \beta, \lambda}^{\mu}+\mathbf{I}_{\sigma \beta}^{\mu} \mathbf{I}_{\alpha \lambda}^{\sigma}-\mathbf{I}_{\sigma \lambda}^{\mu} \mathbf{I}_{\delta \beta}^{\sigma}\right) \boldsymbol{\xi}^{\lambda} \frac{d \mathbf{X}^{\delta}}{d \tau} \frac{d^{2} \mathbf{X}^{\beta}}{d \tau^{2}}
\end{aligned}
$$

By letting:

$$
\begin{gathered}
\mathcal{R}_{\delta \beta \lambda}^{\mu} \stackrel{\wedge}{=} \mathbf{R}_{\delta \lambda, \beta}^{\mu}-\mathbf{R}_{\delta \beta, \lambda}^{\mu}+\mathbf{R}_{\sigma \beta}^{\mu} \mathbf{R}_{\alpha \lambda}^{\sigma}-\mathbf{R}_{\sigma \lambda}^{\mu} \mathbf{R}_{\delta \beta}^{\sigma}, \\
\mathfrak{J}_{\delta \beta \lambda}^{\mu} \stackrel{\wedge}{=} \mathbf{I}_{\delta \lambda, \beta}^{\mu}-\mathbf{I}_{\delta \beta, \lambda}^{\mu}+\mathbf{I}_{\sigma \beta}^{\mu} \mathbf{I}_{\alpha \lambda}^{\sigma}-\mathbf{I}_{\sigma \lambda}^{\mu} \mathbf{I}_{\delta \beta}^{\sigma},
\end{gathered}
$$

which are the real and imaginary parts of the curvature Riemannian tensor we can write equation (3.19) as:

$$
\begin{gathered}
\frac{d^{3} \boldsymbol{\xi}^{\mu}}{d \tau^{3}}+2 \mathbf{R}_{\delta \beta}^{\mu} \frac{d^{2} \mathbf{X}^{\delta}}{d \tau^{2}} \frac{d \boldsymbol{\xi}^{\beta}}{d \tau}+\mathbf{R}_{\delta \lambda, \beta}^{\mu} \boldsymbol{\xi}^{\lambda} \frac{d \mathbf{X}^{\delta}}{d \tau} \frac{d^{2} \mathbf{X}^{\beta}}{d \tau^{2}} \\
+\mathbf{R}_{\sigma \beta}^{\mu} \mathbf{R}_{\alpha \lambda}^{\sigma} \boldsymbol{\xi}^{\lambda} \frac{d \mathbf{X}^{\delta}}{d \tau} \frac{d^{2} \mathbf{X}^{\beta}}{d \tau^{2}}-\mathbf{R} \sigma \lambda^{\mu} \mathbf{R}_{\delta \beta}^{\sigma} \boldsymbol{\xi}^{\lambda} \frac{d \mathbf{X}^{\delta}}{d \tau} \frac{d^{2} \mathbf{X}^{\beta}}{d \tau^{2}} \\
+2 \mathbf{I}_{\delta \beta}^{\mu} \frac{d \mathbf{X}^{\delta}}{d \tau} \frac{d \boldsymbol{\xi}^{\beta}}{d \tau}+\mathbf{I}_{\delta \lambda, \beta}^{\mu} \boldsymbol{\xi}^{\lambda} \frac{d \mathbf{X}^{\delta}}{d \tau} \frac{d^{2} \mathbf{X}^{\beta}}{d \tau^{2}}+\mathbf{I}_{\sigma \beta}^{\mu} \mathbf{I}_{\alpha \lambda}^{\sigma} \xi^{\lambda} \frac{d \mathbf{X}^{\delta}}{d \tau} \frac{d^{2} \mathbf{X}^{\beta}}{d \tau^{2}}-\mathbf{I}_{\sigma \lambda}^{\mu} \mathbf{I}_{\delta \beta}^{\sigma} \boldsymbol{\xi}^{\lambda} \frac{d \mathbf{X}^{\delta}}{d \tau} \frac{d^{2} \mathbf{X}^{\beta}}{d \tau^{2}} \\
=-\left(\mathcal{R}_{\delta \beta \lambda}^{\mu}+\mathfrak{J}_{\delta \beta \lambda}^{\mu}\right) \xi^{\lambda} \frac{d \mathbf{X}^{\delta}}{d \tau} \frac{d^{2} \mathbf{X}^{\beta}}{d \tau^{2}} .
\end{gathered}
$$

Using equation (2.23), the freedom to relabel dummy indices and equations (3.21) and (3.22), we can write equation (3.23) as

$$
\begin{gathered}
\frac{d^{3} \boldsymbol{\xi}^{\mu}}{d \tau^{3}}+2\left(\mathbf{I}_{\delta \beta}^{\mu} \frac{d \mathbf{X}^{\delta}}{d \tau}-\mathbf{R}_{\delta \beta}^{\mu} \mathbf{R}_{\mu \beta}^{\delta} \frac{d \mathbf{X}^{\mu}}{d \tau} \frac{d \mathbf{X}^{\beta}}{d \tau}\right) \frac{d \boldsymbol{\xi}^{\beta}}{d \tau} \\
-\left(2\left(\mathcal{R}_{\delta \beta \lambda}^{\mu}+\mathfrak{J}_{\delta \beta \lambda}^{\mu}\right)+\left(\mathbf{R}_{\delta \beta, \lambda}^{\mu}+\mathbf{I}_{\delta \beta, \lambda}^{\mu}\right)\right) \mathbf{R}_{\delta \mu}^{\beta} \boldsymbol{\xi}^{\lambda} \frac{d \mathbf{X}^{\delta}}{d \tau} \frac{d \mathbf{X}^{\mu}}{d \tau} \frac{d \mathbf{X}^{\delta}}{d \tau}=0,
\end{gathered}
$$

which is the $3^{\text {rd }}$-order geodesic deviation equation. It is subsequently interesting to obtain simultaneously the standard and the higher-order deviation equation from the present argument. 


\section{Conclusions and Perspectives}

In the present paper, we have discussed the implications of an extended complex backward-forward derivative operator in differential geometry.

This form of operator is motivated from non-local-in-time Lagrangian dynamics and in our analysis it takes the special form $\mathbf{D}^{n}=D+\mathcal{D}_{R}^{n}-i \mathcal{D}_{I}^{n}$ where $D=d / d t$,

$\mathcal{D}_{R}^{n}=\frac{1}{2 k !} \sum_{k=1}^{n}\left(1+(-1)^{k}\right) \bar{\tau}^{k} D^{(k+1)}$ and $\mathcal{D}_{I}^{n}=\frac{1}{2 k !} \sum_{k=1}^{n}\left(1-(-1)^{k}\right) \bar{\tau}^{k} D^{(k+1)}$.

Through this paper we limited our analysis to $n=1$ where the EulerLagrange equation is complexified and contains derivative operator up to order 2. In differential equation settings, the Euler-Lagrange equation is equivalent to a higher-order geodesic equation which is complexified and is splitted into three independent differential equations which are respectively factors of $L_{1}^{0}, L_{1}^{2}, L_{1}^{4}$.

It was observed that the metric and the Christoffel symbol are complexified and the geodesic equation up to the first order in $\tau$ is complexified and is splitted into differential equations which are the standard geodesic equation and a $3^{r d}$-order geodesic equation. This approach seems promising as both the standard and the higher-order geodesic equations may be derived simultaneously. Some applications were discussed: within the context of general relativity, it was observed that a relation between jerk, velocity and the Newtonian gravitational potential exist and besides, for the case of a time-independent potential, the Laplace equation is obtained yielding the Schwarzschild solution describing the gravitational field outside the a spherical mass directly without using boundary conditions; as a second application, we have derived the geodesic deviation equation and it was observed that the standard geodesic deviation equation is derived besides a $3^{\text {rd }}$-order derivative geodesic deviation equation. This higher-order deviation equation may have interesting implications in planetary motions. A detailed geometric analysis of higher-order geodesic equations, complex manifolds and their implications in Hermitian differential geometry, twistor geometry and field theory are under progress.

\section{References}

[1] F. Ben Adda and J. Cresson, Quantum derivatives and the Schrödinger equation, Chaos Solitons Fractals, 9, (2004), 1323-1334 
[2] Y. Ciann-Dong, On the existence of complex spacetime in relativistic quantum mechanics, Chaos Solitons Fractals, (2008), 316-331

[3] R. Colistete Jr., C. Leygnac, and R. Kerner, Higher-order geodesic deviations applied to the Kerr metric, Class. Quantum Grav, (2002), 4573-4590

[4] M. Davidson, A study of the Lorentz-Dirac equation in complex spacetime for clues to emergent spacetime, J. Phys.: Conf. Series, Conf.1, (2012), (11 pages).

[5] R. A. El-Nabulsi, Non-standard non-local-in-time Lagrangians in classical mechanics, Qual. Theor. Dyn. Sys., (2014), 149-160

[6] G. Esposito, From spinor geometry to complex general relativity, Int. J. Geom. Meth. Mod. Phys, (2005), 675-731

[7] D.Eberly, Computing geodesics on a Riemannian manifold, Geometric Tools, LLC, 2015

[8] R. S. Herman, Derivation of the geodesic equation and defining the Christoffel symbols, a lecture given at the University of North Carolina Wilmington, March 13, 2008

[9] E. L. Hill, On the kinematics of uniformly accelerated motions and classical magnetic theory, Phys. Rev, (1947), 143-149

[10] W. P. Joyce, Gauge freedom of Dirac theory in complexified spacetime algebra, J. Phys. A: Math. Gen, (2002), 4737-4747

[11] R. Kerner, J. W. von Holten, and R. Colistete Jr, Relativistic epicycles: another approach to geodesic deviations, Class. Quantum Grav, (2001), 4725-4742

[12] N. Koike, The complexifications of pseudo-Riemannian manifolds and anti-Kaehler geometry, SUT J. Math, (2014), 271-295

[13] Z.-Y. Li, J.-L. Fu, and L.-Q. Chen, Euler-Lagrange equation from nonlocal-intime kinetic energy of nonconservative system, Phys.Lett, (2009), 106-109

[14] C. W. Misner, K. S. Thorne, and J. A. Wheeler, Gravitation, W. H. Freeman and Co, San Francisco, 1973

[15] R. Ya. Matsyuk, The variational principle for the uniform acceleration and quasispin in two-dimensional space-time, SIGMA, 4, (2008), 016-027

[16] R. Ya. Matsyuk, Lagrangian analysis of invariant third-order equations of motion in relativistic classical particle mechanics, English transl.: Soviet Phys. Dokl, 30, (1985), $458-460$

[17] E. Nelson, Derivation of the Schrödinger Equation from Newtonian Mechanics, Phys. Rev, (1966), 1079-1085

[18] J. Saucedo and V. M. Villanueva J. A. Nieto, Relativistic top deviation equation and gravitational waves, Phys. Lett, (2003), 175-186

[19] L. Nottale, Fractal Space-Time and Microphysics: Towards a Theory of Scale Relativity, World Scientific, 1993

[20] L. Nottale, The theory of scale-relativity: Non-differentiable geometry and fractal space-time, Computing Anticipatory Systems, CASYS'03-Sixth International Conference (Liege, Belgium, 11-16 August 2003), Daniel M. Dubois Editor, American Institute of Physics Conference Proceedings, (2004), 68-95 
[21] G. O. Okengo, On the complexification of Minkowski spacetime, Africa J. Phys. Sci, (2015), 73-76

[22] R. Penrose, On the twistor description of massless fields, In Proceedings Complex Manifold Techniques In Theoretical Physics, ed. by Lawrence, (1978), 55-91

[23] R. Penrose and M.A. MacCallum, Twistor theory: an approach to the quantization of fields and space-time, Phys. Rep, (1972), 241-316

[24] T. Popiel, Higher-order geodesics in Lie groups, Math.Contr. Sign. Syst, No. 3, (2007), 235-253

[25] T. Roubicek, Calculus of variations, Chap. 17 in Mathematical Tools for Physicists, (Ed. M. Grinfeld), J. Wiley, Weinheim, 2014

[26] S. L. Shapiro and S. A. Teukolsky, Black Holes, White Dwarfs and Neutron Stars, Wile-Interscience, New York, 1983

[27] B. G. Sidharth, Complexified spacetime, Found. Phys. Letts, No. 1, (2003), 91-97

[28] H. Stephani, General Relativity-An Introduction to the Theory of the Gravitation Field, Cambridge University Press, 1982

[29] R. Szoke, Complex structures on tangent bundles of Riemannian manifolds, Math. Ann, (1991), 409-428

[30] J. A. K. Suykens, Extending Newton's law from nonlocal-in-time kinetic energy, Phys. Lett, (2009), 1201-1211

[31] S. Taniguchi, On almost complex structures on abstract Wiener spaces, Osaka J. Math, (1996), 189-206

[32] J. Vines, Geodesic deviation at higher order via covariant bitensors, Gen. Rel. Grav, (2015), 49-65

[33] K. Yano, Concircular geometry I. Concircular transformations, Proc. Imp. Acad. Jap, (1940), 195-200

Rami Ahmad El-Nabulsi

Athens Institute for Education and Research, Mathematics and Physics Divisions, 8 Valaoritou Street,Kolonaki, 10671, Athens, Greece

E-mail: nabulsiahmadrami@yahoo.fr

Received: 7.02 .2016

Accepted: 16.05.2016 